\title{
UNA PROPUESTA PEDAGÓGICA FRENTE A LA LECTURA CRÍTICA DE TEXTOS FILOSÓFICOS EN UNA INSTITUCIÓN EDUCATIVA DEL DEPARTAMENTO DE SANTANDER*
}

Henry Alberto Macías Uribe

\section{Resumen}

Este trabajo es el resultado de una investigación que se hizo con estudiantes del grado undécimo bachillerato del colegio rural Pozo Nutrias Dos, del municipio de San Vicente del Chucuri, del departamento de Santander, Colombia. Consistió en indagar por cómo una propuesta pedagógica favorece la lectura crítica de textos filosóficos en la institución educativa. En ese sentido para su desarrollo se planteó como objetivo general el caracterizar una propuesta pedagógica para la lectura crítica de textos filosóficos en la institución, junto con los objetivos específicos de identificar las prácticas lectoras de los estudiantes de undécimo en la asignatura de Filosofía, indagar por el concepto que tienen de lectura crítica estudiantes del grado undécimo

* Artículo de investigación científica y tecnológica resultado de la investigación: "Una propuesta pedagógica frente a la lectura crítica de textos filosóficos en una institución educativa del departamento de Santander". Caso: Colegio público-rural de San Vicente del Chucuri, Santander. El proyecto se desarrolló en correspondencia con los objetivos de la línea de investigación Pedagogía y Lenguaje, con la orientación de la profesora Sonia Gómez Benitez. Citar como: Macias, H. (2018). Una propuesta pedagógica frente a la lectura crítica de textos filosóficos en una institución educativa del departamento de Santander. Cuadernos de Filosofía Latinoamericana, 39(118), 177-193. DOI: https://doi. org/10.15332/10.15332/s0120-8462.2018.0118.10

* Filósofo, magister en Pedagogía. Docente magisterio. Catedra UIS. Correo electrónico: henrymacias821@gmail.com 
y los docentes de Lenguaje de la institución, y por último, caracterizar los textos filosóficos que permiten el accionar de una propuesta pedagógica frente a la lectura crítica de dichos textos en el aula. En cuanto al aspecto metodológico se recurre al paradigma cualitativo y al diseño de investigación acción en educación dentro del contexto de la maestría en Pedagogía ofertada por la UIS.

Palabras clave: pedagogía, lectura crítica, texto filosófico, pensamiento crítico.

\section{A pedagogical proposal about the critical reading of philosophical texts in an educational institution of Santander}

\section{Abstract}

This work is the result of a research done with eleventh grade students of Pozo Nutrias Dos rural high school in San Vicente del Chucurí municipallity, Santander, Colombia. It consisted of investigating how a pedagogical proposal favors the critical reading of philosophical texts in the educational institution. In this sense, for its development, it was proposed as a general objective to characterize a pedagogical proposal for the critical reading of philosophical texts in the institution and, as specific objectives, to identify the reading practices of eleventh grade students in the subject of Philosophy, to inquire into the concept of critical reading that eleventh grade students and language teachers of the institution have and, finally, to characterize the philosophical texts that allow the action of a pedagogical proposal in reference to the critical reading of philosophical texts in the classroom.

In terms of the methodological aspect, the qualitative paradigm and the design of research action in education are used within the context of the Master's in Pedagogy offered by the UIS.

Keywords: Pedagogy, critical reading, philosophical text, critical thinking. 


\section{Uma proposta pedagógica perante a leitura crítica de textos filosóficos em uma instituição educativa do estado de santander*}

\section{Resumo}

Este trabalho é resultado de uma pesquisa feita com estudantes do último grado do ensino médio do colégio rural Pozo Nutrias Dos, do município de San Vicente del Chucuri, no estado de Santander, Colômbia. Consistiu em indagar como uma proposta pedagógica favorece a leitura crítica de textos filosóficos na instituição educativa. Neste sentido para seu desenvolvimento se apresentou como objetivo geral caracterizar uma proposta pedagógica para a leitura crítica de textos filosóficos na instituição, junto com os objetivos específicos para identificar as práticas leitoras dos estudantes de undécimo na disciplina de Filosofia, indagar pelo conceito que tem de leitura crítica os estudantes de undécimo e os docentes de Linguagem da instituição. Por último, caracterizar os textos filosóficos que permitem o agir de uma proposta pedagógica perante a leitura crítica de estes textos na sala de aula. No relacionado ao aspecto metodológico, recorre-se ao paradigma qualitativo e ao desenho de pesquisa ação em educação dentro do contexto do mestrado em Pedagogia oferecido pela UIS.

Palavras-chave: pedagogia, leitura crítica, texto filosófico, pensamento crítico.

\section{Introducción}

La importancia de este estudio se sustentó en que una gran mayoría de estudiantes del país presenta serias dificultades en las competencias lectoras (Durán, 2007), tal como lo demuestran las pruebas tanto nacionales (Duarte, 2012) como internacionales

* Artigo de pesquisa científica e tecnológica resultado da pesquisa: "Uma proposta pedagógica perante a leitura crítica de textos filosóficos em uma instituição educativa do estado de Santander". Caso: Colégio público-rural de San Vicente del Chucuri, Santander. 0 projeto se desenvolveu em concordância com os objetivos da linha de pesquisa Pedagogia e Linguagem, com a orientação da professora Sônia Gómez Benitez. 
(Carabaña, 2008) en las que han participado. Lo anterior, está ligado también a bajos resultados de otras pruebas y asignaturas que, de igual modo, se basan en competencias lectoras, tal como es el caso de la asignatura de Filosofía (Celis, 2010). De modo que se presenta un efecto dominó.

Con base en lo anterior, en un primer momento se caracterizaron las competencias de lectura de los estudiantes de una institución educativa pública del departamento de Santander, luego, se indagó acerca de las concepciones que tienen los estudiantes en relación con la lectura de textos filosóficos y cómo trabajan los diferetes niveles de lectura. Finalmente, se buscó fortalecer la lectura crítica de textos filosóficos a través de una propuesta pedagógica para los estudiantes.

De igual modo, se esperó que los estudiantes tomaran conciencia de sus prácticas lectoras, con el fin de que entiendan lo importante que es para su futuro desarrollo profesional el buen manejo de la competencia lectora. Así mismo, a corto plazo, la expectativa fue realizar una lectura crítica con las exigencias propias que demanda un texto filosófico. Por último, la institución se benefició, en tanto se fomentó la formación de estudiantes conscientes del valor de fortalecer la competencia lectora, no solo en la asignatura de Filosofía y Lenguaje, sino, por ejemplo, en el área de Ciencias Naturales, entre otras, que implicó una transformación positiva de la comunidad educativa en general.

En lo que refiere al impacto social, los estudiantes que asumieron el papel de ser lectores críticos, sin duda serán ciudadanos críticos y autónomos, que reflexionan sobre sí mismos y sobre su contexto social, siendo gestores de cambio tanto de su comunidad educativa como de la sociedad.

Por último, se aportó referentes para reestructurar el trabajo de aula en la asignatura de Filosofía, con base en el carácter interdisciplinario de las competencias lectoras.

\section{Referentes teóricos}

\section{El rol del docente mediador en el fomento de la lectura crítica}

El papel tradicional del docente como aquel que transmite la verdad es cosa del pasado, atrás quedó esa figura del maestro medieval que a cada paso que daba tenía un alumno, sin luz, ávido de conocimiento detrás de él. El papel del docente hoy 
es más bien el de un orientador en un proceso educativo en el que este gestiona los aprendizajes y reconstruye saberes, lo que implica además que la perspectiva epistemológica concibe el saber ya no como algo acabado y que está ahí para descubrir, tal como lo plantearía en su momento el paradigma positivista (Ayer, 1965), sino que se entiende el conocimiento como algo inacabado en el sentido que lo plantea Popper (1985), por lo que la indagación por el saber ha de ser permanente. En términos constructivistas, el conocimiento es una creación con un sujeto activo, creador (Arceo y Rojas, 2002). En este sentido el perfil del educador es idóneo en cuanto cumpla con ciertas características, ya que desde esta perspectiva es importante la adquisición de aprendizajes significativos, a partir de estrategias facilitadoras del proceso en las que se plantean las situaciones de aprendizaje pertinentes: el educador es facilitador de estas.

El modelo del constructivismo concibe la enseñanza como una actividad crítica (Porlán y Ariza, 1993), donde no es una simple transmisión de conocimiento, sino también de métodos que permiten al estudiante construir su propio saber, por lo que resulta un modelo pedagógico pertinente para fomentar la lectura crítica.

Así mismo, para este trabajo resultó importante la relación de los componentes de asimilación y acomodación con relación al proceso de la lectura que el estudiante con su pensamiento lógico formal realiza en forma constructiva (Piaget, 2008). A través de las estrategias el constructivismo asume que el aprendizaje es un proceso dinámico que implica siempre una reconceptualización del conocimiento. Por tanto, se destaca que en el proceso evaluativo las preguntas o situaciones deben buscar que el estudiante exprese con sus propias palabras lo aprendido. Pues, hay que considerar que si el estudiante es capaz de expresar coherentemente lo aprendido, está en condiciones de sostener que aprendió significativamente (Ortega et al., 1993). Caso contrario, si se acepta que el estudiante repita tal y como se le enseña, o tal y como aparece en los textos, se presenta una lectura literal, dicha mecánica no lleva al educando a asumir juicios de valor acerca de su proceso lector.

De igual manera la perspectiva constructivista enfatiza en que las actividades de enseñanza y las actividades evaluativas deben justificarse con respecto a su pertinencia o provecho para el estudiante (Carretero, 2000). Esto plantea de alguna manera la idea de que los contenidos deben apuntar al desarrollo de competencias significativas que le sirvan al educando para enfrentar exigencias cognitivas reales, por lo que las prácticas evaluativas que enfatizan la memorización mecánica de fórmulas, fechas y la transcripción de libros, son incongruentes con la propuesta constructivista. 
Llegados a este punto es importante enfatizar en la idea de que la lectura crítica no tiene que ver con una lectura mecánica (Freire, 1999) y un proceso de memorización, sino con un proceso complejo y constructivo en el que se deben poner en práctica diferentes habilidades, tales como: descifrar el texto, contextualizar los conceptos o ubicarlos en sus ambientes correspondientes, relacionar información, extrapolarla o lograr orientarla hacia las diferentes situaciones contextúales pertinentes, de tal manera que el texto se pueda valorar con su real y total significado construido (Carol, 2008).

El estudiante debe poner en juego el conocimiento previo que posee sobre el contenido del texto y relacionar su concepción actual de la lectura, se puede decir que el estudiante tiene la misión en esta estrategia de reconstruir el significado del texto y además está en condiciones de expresarlo.

En ese sentido, para Vygotsky el aprendizaje no se da fuera de la Zona de Desarrollo Próximo (ZDP), esta es la distancia entre el nivel de desarrollo determinado por la capacidad de resolver independientemente un problema y el nivel de desarrollo potencial, determinado a través de la resolución de un problema, bajo la guía de un adulto o en colaboración con otro sujeto más capacitado (Vygotsky, 2000). En el terreno educativo se puede señalar cómo se conducen a enfocar la atención en las habilidades o capacidades ya constituidas y dominadas por el educando, sin decir nada acerca de lo que está en proceso de aprenderse, pero que por el momento solo se puede realizar conjuntamente con otra persona que es más experta en la tarea o el problema en cuestión, es decir, un maestro mediador. Para Ausubel el conocimiento que se transmite en cualquier situación de aprendizaje debe estar estructurado no solo en sí mismo, sino con respecto al conocimiento que ya posee el estudiante, por lo que la organización y secuenciación de contenidos docentes deben tener en cuenta esos conocimientos previos (Ausubel, Novak y Hanesian, 1983). Con todo lo dicho líneas atrás, para el docente el aprendizaje debe ser una actividad significativa, lo que se traduce en que la persona aprenda y además que ese conocimiento se relacione, como se señaló líneas atrás, con los conocimientos previos (Torres, 2003).

\section{El pensamiento critico desde Boisvert}

Para el diseño y la aplicación de la propuesta de investigación se consideró la concepción de pensamiento crítico de Boisvert por ser el autor fundamental para llevar a buen término el proceso de la lectura crítica, porque desde su perspectiva y la reflexión acerca de sus planteamientos nos llevan a pensar lo difícil que es alimentar 
el pensamiento crítico sin la lectura, y aún más, hacer lectura crítica sin pensamiento crítico.

Para Boisvert el pensamiento crítico como proceso y ejercicio en forma estructurada, se piensa desde que aparece un problema, pregunta difícil, estado de duda, circunstancias que producen perplejidad en el individuo, y cuyas características complementarias para el pensamiento crítico son: amplitud mental, honestidad intelectual junto con las capacidades de razonamiento y de investigación lógica (Boisvert, 2004; Popper, 1985). Actitudes que posibilitan el pensamiento crítico como factor para la resolución de problemas. En el contexto de la lectura sería reconocer qué es lo que el texto dice tanto explícita como implícitamente, de modo que se identifican los diferentes componentes textuales. Tal como se expresa en la figura 1.

Figura 1. El pensamiento crítico como proceso

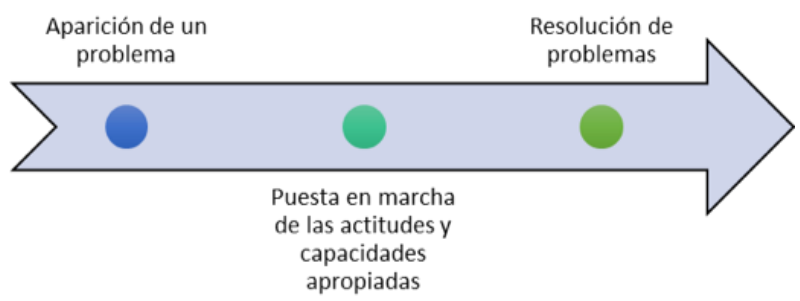

Fuente. Elaboración propia basado en el planteamiento de Boisvert

El pensamiento crítico comprende analizar y dominar las innumerables informaciones que caracteriza el contexto social actual. En cuanto a la función del pensamiento crítico dentro de las instituciones educativas, es así que toda verdadera enseñanza debe incluir la formación en pensamiento crítico en el sentido de que aspire a favorecer la autonomía del estudiante (Boisvert, 2004). Además, dentro de las muchas bondades que puedan existir, el ejercicio del pensamiento crítico aplicado a la comunicación permite: delimitar con precisión un problema, ordenar ideas, expresarlas con coherencia, obtener conclusiones y evaluarlas por lo que la importancia del pensamiento crítico radica en conocer y comprender las razones que tienden a la finalidad educativa, es decir, formar la agudeza de alguien significa desarrollar sus aptitudes intelectuales, así que favorecer el desarrollo de las facultades intelectuales 
de las personas desde el pensamiento crítico es un fin importante de la educación (Boisvert, 2004). En la tabla 1 se presentan las fases del pensamiento crítico desde los planteamientos de Boisvert.

Tabla 1. Fases del pensamiento crítico

\begin{tabular}{|l|l|l|}
\hline $\begin{array}{c}\text { Primera fase. Habilidades } \\
\text { del pensamiento }\end{array}$ & $\begin{array}{c}\text { Segunda fase. Reflexión } \\
\text { sobre el tema o contenido } \\
\text { disciplinario }\end{array}$ & $\begin{array}{l}\text { Tercera fase. Aplicación de } \\
\text { las habilidades y procesos } \\
\text { de pensamiento }\end{array}$ \\
\hline $\begin{array}{l}\text { Definir habilidades precisas. } \\
\text { Enseñarlas de manera } \\
\text { explícita con ejercicios en } \\
\text { los que el estudiante precise } \\
\text { qué es lo que más le impacta } \\
\text { del conocimiento y dialogue } \\
\text { con el objeto de estudio. }\end{array}$ & $\begin{array}{l}\text { Alcanzar aprendizaje más } \\
\text { allá de una evaluación. }\end{array}$ & $\begin{array}{l}\text { Aplicación de las } \\
\text { habilidades y procesos de } \\
\text { pensamiento a diversas } \\
\text { situaciones del contexto } \\
\text { escolar y vida cotidiana. }\end{array}$ \\
\hline $\begin{array}{l}\text { Objetivo: aprender a } \\
\text { validar lo que se conoce } \\
\text { extrapolando a diferentes } \\
\text { campos de conocimiento. }\end{array}$ & $\begin{array}{l}\text { Objetivo: comprender } \\
\text { los problemas que se dan } \\
\text { en una situación a través } \\
\text { del diálogo con otros o } \\
\text { el descubrimiento por sí } \\
\text { mismo. }\end{array}$ & $\begin{array}{l}\text { Objetivo: reflexión } \\
\text { metacognitiva, ir más allá de } \\
\text { la información literal siendo } \\
\text { consciente de su propio } \\
\text { proceso de pensamiento } \\
\text { para controlarlo y mejorar } \\
\text { su forma de pensar. }\end{array}$ \\
\hline $\begin{array}{l}\text { Actividad: Clasificar y } \\
\text { comparar. }\end{array}$ & $\begin{array}{l}\text { Actividad: aprendizaje } \\
\text { cooperativo, uso de } \\
\text { organizadores gráficos. }\end{array}$ & $\begin{array}{l}\text { Actividad: autoevaluación y } \\
\text { autocorrección para corregir } \\
\text { errores de manera asertiva. }\end{array}$ \\
\hline
\end{tabular}

Fuente. Elaboración propia con base en el trabajo de grado de Luis Alfredo Mantilla titulado: "Propuesta pedagógica para hacer de la clase de Lengua Castellana un espacio generador de pensamiento crítico"

\section{Niveles de lectura}

Los niveles de lectura, que hacen parte de lo que llama Pérez Abril, dimensión técnica y cognitiva de la lectura se usan para evaluar a los estudiantes colombianos en las pruebas nacionales (Pérez, 2003).

Existe una división de los niveles de lectura establecidos por el ICFES en la prueba de lenguaje que son tres: literal, inferencial y crítico. 
En lo que refiere al nivel literal, según el ICFES, se explora lo que aparece en el texto de modo explícito. Se privilegia el sentido literal de la palabra y su función dentro de una oración o de un párrafo. De modo que se extrae la información sin agregar valores interpretativos. Dentro de la comprensión literal, se recupera la información explícitamente planteada en el texto y se la reorganiza mediante clasificaciones, resúmenes y síntesis.

El nivel inferencial consiste en relacionar información del texto con la información que no aparece de modo explícito, lo que supone una comprensión del texto a partir de la cual el lector hace inducciones o deducciones, además de reconocer la intención comunicativa del texto. Se trata, en últimas, de buscar relaciones que están más allá del texto, en donde se relaciona la nueva información con los saberes previos a través de los cuales el lector formula hipótesis.

Por último, el nivel de lectura crítica consiste en una toma de posición argumentada del lector frente al texto. En donde este relaciona el nuevo saber con su presaber. De modo que es necesario analizar la intención de los textos y los autores, además de ideologías que puedan estar presentes. Por lo que el lector al evaluar el contenido desde su posición, reconoce y sopesa a la vez el texto. Por último, el nivel de lectura crítica comprende el nivel literal e inferencial de lectura (Instituto Colombiano para el Fomento de la Educación Superior - ICFES, 2016).

\section{Metodología}

La metodología tiene un enfoque cualitativo que se caracteriza por explorar los fenómenos en profundidad, se conduce en ambientes naturales y los significados se extraen de los datos y no se fundamenta en la estadística. Además, es un proceso inductivo, en el que a partir de un caso particular se llega a conclusiones generales, recurrente y donde se analizan múltiples realidades subjetivas. Con relación a las bondades se tiene que la profundidad de significados, amplitud, riqueza interpretativa, además de que contextualiza el fenómeno no tiene secuencia lineal (Sampieri, Collado y Lucio, 2014). El enfoque cualitativo consiste:

En la búsqueda cualitativa, en lugar de iniciar con una teoría y luego "voltear" al mundo empírico para confirmar si esta es apoyada por los datos y resultados, el investigador comienza examinando los hechos en sí y en el proceso desarrolla 
una teoría coherente para representar lo que observa. Dicho de otra forma, las investigaciones cualitativas se basan más en una lógica y proceso inductivo (explorar y describir, y luego generar perspectivas teóricas). Van de lo particular a lo general. Por ejemplo, en un estudio cualitativo típico, el investigador entrevista a una persona, analiza los datos que obtuvo y saca conclusiones; posteriormente, entrevista a otra persona, analiza esta nueva información y revisa sus resultados y conclusiones; del mismo modo, efectúa y analiza más entrevistas para comprender el fenómeno que estudia. Es decir, procede caso por caso, dato por dato, hasta llegar a una perspectiva más general. (Sampieri, Collado y Lucio, 2014, p. 8)

En este trabajo se considera el enfoque cualitativo porque se quiere llegar a la comprensión de un fenómeno social ligado a la lectura crítica de textos filosóficos, a partir del manejo de información para la elaboración de hallazgos, ya que "con el término "investigación cualitativa", se entiende cualquier tipo de investigación que produce hallazgos a los que no se llega por medio de procedimientos estadísticos u otros medios de cuantificación" (Strauss, Corbin y Zimmerman, 2002). Como docentes de aula, se asume el rol de investigador por contar con acceso al ambiente natural, donde se desarrolla la investigación con lo cual el fenómeno al ser estudiado será conocido e interpretado en el espacio y tiempo en el que se desarrolla cotidianamente, sin forzar ni provocar hechos o situaciones que rompan con esa naturalidad. Pues, el contexto es parte de la información y el ambiente influye en el tipo de dato que se va a construir (Denzín y Lincoln, 2012).

El diseño metodológico es Investigación-Acción (IA) y se considera pertinente para esta propuesta, porque permite ayudar en la búsqueda de planes posibles y la formulación de soluciones al problema de investigación, de igual manera la IA propicia el cambio social y como transformación de la realidad, las personas tienen un papel en ese proceso de cambio (Mckernan, 1999). Para Sandín (2003), en el contexto educativo permite observar situaciones de aula, y como docente investigador evidenciar cómo los estudiantes realizan su proceso de escritura, por lo que:

La investigación-acción se encuentra ubicada en la metodología de investigación orientada a la práctica educativa. Desde esta perspectiva, la finalidad esencial de la investigación no es la acumulación de conocimientos sobre la enseñanza o la comprensión de la realidad educativa, sino, fundamentalmente, aportar 
información que guíe la toma de decisiones y los procesos de cambio para la mejora de esta. Justamente, el objetivo prioritario de la investigaciónacción consiste en mejorar la práctica en vez de generar conocimientos; así, la producción y utilización del conocimiento se subordina a este objetivo fundamental y está condicionado por él. (Sandín, 2003, p. 161)

La IA es el estudio de una situación social para tratar de mejorar la calidad de la acción. Su objetivo consiste en proporcionar elementos que sirvan para facilitar el juicio práctico en situaciones concretas. La validez de las teorías e hipótesis que genera no depende tanto de pruebas "científicas" del concepto de la verdad, sino de su utilidad para ayudar a las personas a actuar de modo más inteligente y acertado (Elliott, 1990). En la IA las "teorías" no se validan de forma independiente para aplicarlas luego a la práctica, sino que lo hacen a través de la práctica. Además, la IA posibilita la resolución de problemas en el aula en la medida que los docentes estén en constante formación, con el fin de implementar nuevos enfoques que resulten innovadores dentro de un contexto de enseñanza-aprendizaje, todo para transformar y mejorar la educación y la sociedad. Por último, pese a que en rasgos generales la investigación acción se desarrolla siguiendo un modelo en espiral, es decir, en ciclos sucesivos que incluyen diagnóstico, planificación, acción, observación y reflexiónevaluación y que el proceso de investigación acción es descrito con matizaciones diferentes según autores, variando en cuanto a su complejidad (Herreras, 2004), es de aclarar que para esta investigación se considera el modelo propuesto por Elliot donde se enfatizan dos importantes características de la IA, a saber, la teoría y la práctica. La siguiente figura ilustra el modelo de Elliot. 
Figura 2. Modelo de Elliot IA

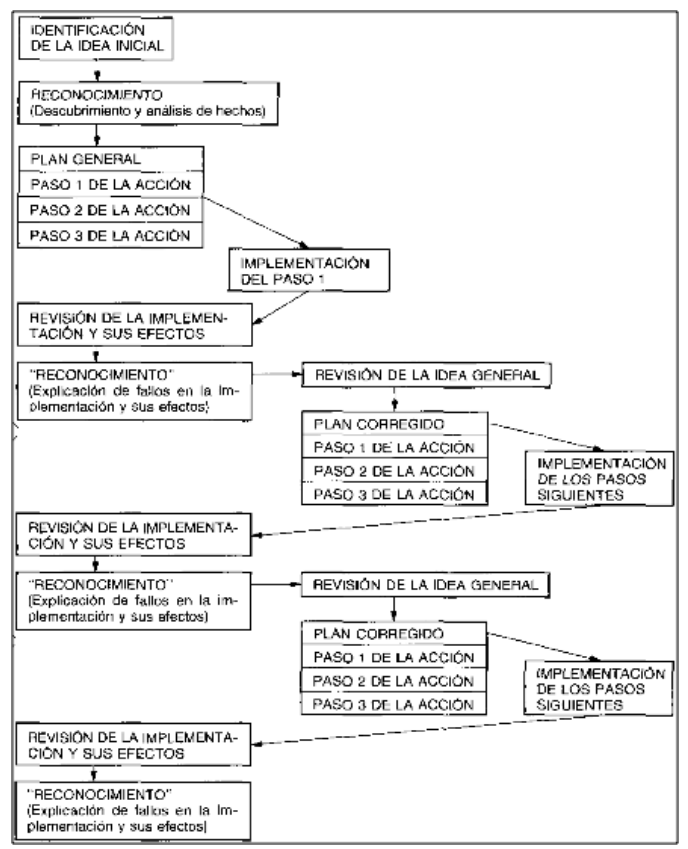

Fuente. Elliot, John. El cambio educativo desde la Investigación-Acción. 3. a ed. Madrid, Morata, 2000, p. 90

\section{Participantes y contexto}

En esta investigación participaron 18 estudiantes de undécimo grado, conformado por 9 hombres y 9 mujeres, con edades comprendidas entre los 14 hasta los 18 años. Los padres de familia manejan un nivel de escolaridad que va desde primaria, en su gran mayoría, hasta un caso de un padre que hizo carrera universitaria.

\section{Procedimiento}

En la siguiente tabla se evidencia cómo fue el procedimiento que se llevó durante la investigación, con sus respectivas fases, objetivos, técnicas e instrumentos. 


\begin{tabular}{|c|c|c|c|}
\hline Fases & Objetivos & Técnicas & Instrumentos \\
\hline $\begin{array}{l}\text { 1. Identificación de } \\
\text { una idea general. } \\
\text { Elaboración del } \\
\text { plan. }\end{array}$ & $\begin{array}{l}\text { 1. Identificar las } \\
\text { prácticas lectoras } \\
\text { de los estudiantes } \\
\text { de undécimo en la } \\
\text { asignatura de Filosofía. } \\
\\
\text { 2. Indagar por el } \\
\text { concepto que tienen } \\
\text { de lectura crítica } \\
\text { estudiantes del grado } \\
\text { undécimo y los } \\
\text { docentes de secundaria } \\
\text { de la Institución } \\
\text { Educativa Pozo Nutrias } \\
\text { Dos. }\end{array}$ & $\begin{array}{l}\text { Entrevista } \\
\text { estructurada } \\
\text { Se realizó con los } 18 \\
\text { estudiantes. } \\
\\
\text { Grupo focal } \\
\text { Se realizó con los } 18 \\
\text { estudiantes y } 1 \text { docente } \\
\text { de Lengua Castellana. }\end{array}$ & $\begin{array}{l}\text { Guía de la entrevista. } \\
\text { Guía de la entrevista. }\end{array}$ \\
\hline $\begin{array}{l}\text { 2. Exploración de } \\
\text { acciones que hay } \\
\text { que realizar para } \\
\text { cambiar la práctica. } \\
\text { Marcha del plan. }\end{array}$ & $\begin{array}{l}\text { 3. Caracterizar los } \\
\text { textos filosóficos que } \\
\text { permiten el accionar } \\
\text { de una propuesta } \\
\text { pedagógica frente a la } \\
\text { lectura crítica de textos } \\
\text { filosóficos en el aula. }\end{array}$ & $\begin{array}{l}\text { Taller investigativo. } \\
\text { Observación partici- } \\
\text { pante. }\end{array}$ & $\begin{array}{l}\text { Guía del taller. } \\
\text { Diario de campo. }\end{array}$ \\
\hline $\begin{array}{l}\text { 4. Evaluación del } \\
\text { plan. }\end{array}$ & $\begin{array}{l}\text { - La evaluación. } \\
\text { - La revisión del plan } \\
\text { general. }\end{array}$ & Grupo focal. & $\begin{array}{l}\text { Grabación } \\
\text { guía del grupo. }\end{array}$ \\
\hline
\end{tabular}

Fuente. Elaboración propia

\section{Análisis de datos}

Se realizó el análisis de los datos obtenidos gracias a las técnicas e instrumentos de manera cualitativa, con el fin de proponer una interpretación a los objetivos de investigación que finalmente permitiera implementar una propuesta pedagógica para la lectura crítica de textos filosóficos.

\section{Resultados}

Para la realización de esta investigación se llevó a cabo un proceso de recolección, selección y sistematización de datos, con el fin de ofrecer una interpretación tanto al problema de investigación como a los objetivos planteados. Por lo cual, además, se planteó una relación entre las fases, objetivos, técnicas e instrumentos. 
Con relación al objetivo general de esta investigación se encontró que al caracterizar una propuesta pedagógica para la lectura crítica de textos filosóficos en una institución educativa del departamento de Santander, resultó importante estructurarla en ocho talleres, los cuales se caracterizan en su contenido por estar acorde, en un primer momento, con los lineamientos establecidos por el ICFES acerca de lo que es la competencia de la lectura crítica.

Otra de las características de la propuesta pedagógica fue que los talleres contaran con una estructura en su orden expositivo, es por eso que en cada uno de estos se presenta el tema, objetivo general, objetivos específicos, recursos, estrategias de enseñanza a implementar (Arceo y Rojas, 2002), además, en los talleres se incluye una etapa de preparación, etapa de enseñanza, etapa práctica y una posterior evaluación con prueba de selección múltiple similar a las que presentan en las pruebas saber.

\section{Conclusiones}

- El ejercicio de lectura crítica de un texto filosófico que se lleve al aula debe tener características tanto formales como de contenido, y que resulten llamativas para el estudiante. Idealmente, que los contenidos estén relacionados con su contexto social y el aspecto formal no se vuelva tortuoso, ya sea porque el texto es oscuro o de difícil comprensión en sí mismo, o porque la terminología que lo compone resulta muy distante del léxico estudiantil.

- El ejercicio de lectura crítica debe partir, en primer lugar, de una idea clara de lo que es la lectura crítica y bajo qué aspectos se puede formar a un lector crítico. Entendiendo además que la lectura crítica no es un ejercicio que se limita al ámbito de las humanidades, y que por tanto es solo responsabilidad de los docentes de humanidades, sino que es una competencia de carácter interdisciplinar y que por tal razón es fundamental preguntarse cómo desde las diferentes áreas del conocimiento se puede fomentar la lectura crítica.

- Implementar propuestas pedagógicas en cuanto a la lectura crítica, que además impliquen un aprendizaje significativo de los estudiantes resulta fundamental para que ellos a su vez entiendan que la lectura crítica es una competencia no solo para satisfacer unas calificaciones que se le pueden dar en el colegio, sino que tiene que ver con una competencia para su desarrollo como ciudadano. 
- Un lector crítico es ante todo un lector, es decir, tiene hábitos lectores, por lo tanto, es importante que la institución educativa parta de la importancia de fomentar mejores hábitos lectores en los estudiantes, que por cierto poco o nada leen, ni por tareas asignadas ni por gusto, ni siquiera leen los subtítulos de las películas. En ese sentido también la institución puede involucrar a toda la comunidad educativa, como por ejemplo los padres de familia.

- Las estrategias pedagógicas para la lectura crítica en el aula deben ser acordes con contenidos implícitos y explícitos de un texto. Los ejercicios de lectura crítica deben ir encaminados a que reconozca la intencionalidad del autor, el propósito, incluso que se logren identificar las ideologías que pueda tener un texto y a su vez confrontarlas con las propias ideologías que se toman como lector. Importante que toda estrategia pedagógica vaya enfocada al aprendizaje colaborativo y por supuesto indague por los presaberes de los estudiantes.

\section{Referencias}

Adorno, T. W. (1962). Prismas: la crítica de la cultura y la sociedad. Ariel.

Arceo, F. D., y Rojas, G. H. (2002). Estrategias docentes para un aprendizaje significativo: una interpretación constructivista. McGraw-Hill.

Ausubel, D. P., Novak, J. D., y Hanesian, H. (1983). Psicología educativa: un punto de vista cognoscitivo. Trillas.

Ayer, A. J. (1965). El positivismo lógico. Fondo de Cultura Económica.

Boisvert, J. (2004). La formación del pensamiento crítico: teoría y práctica. Fondo de Cultura Económica.

Carabaña, J. (2008). Las diferencias entre países y regiones en las pruebas PISA. Documentos de Trabajo del Colegio Libre de Eméritos, 2. Recuperado de http:// www.actiludis.com/wp-content/uploads/2009/09/pisa_carabana_vf.pdf

Carretero, M. (2000). Constructivismo y educación. Editorial Progreso.

Celis, I. (2010). Estrategias creativas para el desarrollo de habilidades de comprensión lectora. Universidad Rafael Belloso Chacín. Maracaibo. 
Denzín, N. K., y Lincoln, Y. S. (2012). Manual de investigación cualitativa (vol. 1). Gedisa. Recuperado de http://www.ceil-conicet.gov.ar/wp-content/ uploads/2015/10/Denzin-Intro-Handbok.rtf

Duarte, J., Bos, M. S., y Moreno, M. (2012). Calidad, igualdad y equidad en la educación colombiana (análisis de la prueba SABER 2009). InterAmerican Development Bank. Recuperado de https:/publications.iadb.org/ handle/11319/5425?locale-attribute $=$ es $\&$

Durán, J. G., y Emiluz, L. (2007). Desarrollo de competencias lectoras a partir de un programa de acompañamiento en estudiantes de educación básica del municipio de Galapa (Atlántico). Barranquilla: Fundación Universidad del Norte.

Elliott, J. (1990). La investigación-acción en educación. Ediciones Morata.

Esteban, M. P. (2003). Investigación cualitativa en educación: fundamentos y tradiciones. McGraw-Hill.

Freire, P. (1999). La importancia de leer y el proceso de liberación. Siglo XXI.

Goodman, K. (2015). Sobre la lectura: una mirada de sentido común a la naturaleza del lenguaje y la ciencia de la lectura.

Habermas, J. (1981). Historia y crítica de la opinión pública: la transformación estructural de la vida pública. Gustavo Gili.

Habermas, J., y Espí, C. M. (2008). Teoría y praxis: estudios de filosofía social. Tecnos.

Herreras, E. B. (2004). La docencia a través de la investigación-acción. Revista Iberoamericana de Educación. Recuperado de https://rieoei.org/RIE/article/ view/2871/3815

Kincheloe, J. L. (2001). Hacia una revisión crítica del pensamiento docente. Octaedro.

Laveaga, J. R. (s.f.). Bases de la investigación cualitativa. Técnicas y procedimientos para desarrollar la Teoría Fundamentada. Recuperado de https://www.academia. edu/949983/Bases_de_la_investigaci\%C3\%B3n_cualitativa._T\%C3\%A9cnicas_y_ procedimientos_para_desarrollar_la_Teor\%C3\%ADa_Fundamentada

McKernan, J. (1999). Investigación-acción y curriculum: métodos y recursos para profesionales reflexivos. Ediciones Morata. 
Ortega, E. M., Salvador, C. C., Majós, T. M., Gallart, I. S., Mestres, M. M., Vidiella, A. Z., y Goñi, J. O. (1993). El constructivismo en el aula. Grao.

Palmero, M. L. (2008). La teoría del aprendizaje significativo en la perspectiva de la psicología cognitiva. Editorial Octaedro, S. L.

Pérez, M. (2003). Leer y escribir en la escuela. Bogotá: ICFES.

Piaget, J., y Inhelder, B. (2008). Psicología del niño. Ediciones Morata.

Popper, K. R. (1985). La lógica de la investigación científica. Tecnos.

Popper, K. R. (2008). Sociedad abierta, universo abierto: Conversación con Franz Kreuzer. Tecnos.

Porlán, R., y Ariza, R. P. (1993). Constructivismo y escuela: hacia un modelo de enseñanza-aprendizaje basado en la investigación. Diada Editoras, S. L.

Sampieri, R. H., Collado, C. F., y Lucio, P. B. (2014). Metodología de la investigación. McGraw-Hill Education.

Smith, F. (1983). Comprensión de la lectura: análisis psicolingüístico de la lectura y su aprendizaje. Trillas.

Sandín, M. P. (2003). Investigación cualitativa en educación. Fundamentos y tradiciones. Madrid. McGraw-Hill.

Strauss, A. L., Corbin, J., y Zimmerman, E. (2002). Bases de la investigación cualitativa: técnicas y procedimientos para desarrollar la teoría fundamentada. Medellín, Colombia: Universidad de Antioquia.

Torres, M. (2003). La Lectura. Factores y Actividades que Enriquecen el Proceso. Revista Educere, 6(20), 389-396. Merida: Universidad de los Andes.

Valls, R., Soler Gallart, M., y Flecha, R. (2008). Lectura dialógica: interacciones que mejoran y aceleran la lectura. Revista Iberoamericana de Educación (OEI), 46, 71-87. Recuperado de http://diposit.ub.edu/dspace/handle/2445/58596

Vygotsky, L. S. (2000). El desarrollo de los procesos psicológicos superiores. Grupo Planeta (GBS). 
